\title{
USE OF THE ANALYTIC HIERARCHY PROCESS IN MAJOR LEAGUE BASEBALL
}

\author{
Lawrence Bodin \\ Robert H. Smith School of Business \\ University of Maryland \\ College Park, MD 20742 \\ Email: lbodin@rhsmith.umd.edu
}

\begin{abstract}
Every year, there are over 100 free agents in Major League Baseball. Each free agent can negotiate a contract with any of the 30 major league teams. After the 1998 season, Kevin Brown signed the largest free agent contract ever $-\$ 105$ million over 7 years. Using the Kevin Brown situation as an example, one can see that a free agent contract is characterized by both the number of dollars paid to the player and the term of the contract. In this paper, a ratings model using the Analytic Hierarchy Process (AHP) is proposed to rank the free agents in Major League Baseball. As such, the AHP tree is displayed and our highest rated agents is shown. The paper concludes with an analysis of the results of this study.
\end{abstract}

\section{Introduction}

Free agency has existed in baseball for over 20 years. Baseball players who qualify as free agents can sell their services to the 'highest bidder.' It is commonplace for key players on a team in one season to become free agents and move to other teams the following season. Generally, over 100 players are free agents each year. After the 1998 baseball season was completed, about 128 players declared for free agency. The rules for free agency are quite complex. To be declared a free agent, a player has to have completed his current contract and have a minimum number of years playing Major League Baseball (MLB). Most free agents are at least 30 years old and have played for at least 4 years. Signing free agents can be a risky and expensive operation that involve signing older players to long term and high dollar contracts.

There can be an additional cost to a team that signs a free agent over and above the salary paid to a free agent,. Under the rules of MLB, some free agents can be signed without compensating the teams that the player played for the previous year while other free agents require compensation, generally in the form of draft choices in the annual high school and college player draft. The high school and college player draft allows each team to select students in high school and college that meet specified regulations. Only a small percentage of players selected in the high school and college draft ever play for a major league baseball team. It generally takes at least two years in the minor league for any of these players to make a major league team.

For 1999, the payroll for the players on the New York Yankees and the Baltimore Orioles is over $\$ 80$ million whereas the payroll for the players on several other teams is under $\$ 25$ million. It is becoming increasing more difficult for teams to be successful at the highest level without incurring very high salary costs. The salaries for the four teams that made the semifinals in the 1997 playoffs were among the five teams with the highest salaries. No team in 1998 with a total salary less than $\$ 42$ million qualified for the playoffs. As the Baltimore Orioles found out in 1998, signing many expensive free agents is not a guarantee of making the players. However, it is apparent that not investing in free agents almost guarantees that a team will not make the playoffs. This issue of 
the lack of parity between the teams is a major concern for MLB and has invoked much discussion in the last couple of years.

It is, therefore, apparent that a team has to be very judicious in deciding upon the free agents to attempt to sign to a contract. Contracts can involve millions of dollars of salary spread out over the term of the contract (generally 1-7 years). It would appear that the use of some type of model to rank the players could be of use to a major league team. In this paper, a model based on the Analytic Hierarchy Process (AHP) is proposed for allowing a MLB team to rank the available free agents. AHP has been successfully used to rank players for an existing MLB team in the 1997 MLB expansion draft. The analysis of the use of AHP to rank the players for the San Diego Padres baseball team for the 1997 expansion draft can be found in Bodin and Epstein 1999.

\section{The AHP Model}

The sideway view for the AHP tree for the ranking of the free agent players is shown in Figure 1. Outside of the Age of the Player, all of the other criteria used in this model can be regarded as subjective criteria. That is to say, we did not use quantifiable criteria such as a player's batting average, steals, home runs, earned run average, wins and saves. These findings are similar to the findings in Bodin and Epstein 1999. Bodin and Epstein 1999 found that subjective criteria were easier to use, more flexible and very effective.

As in Bodin and Epstein 1999, the AHP model that we build is a ratings model. As such, there are no direct pairwise comparisons between players. Instead, pairwise comparisons are carried out at each level of the tree shown in Figure 1 and a ratings model is used to carry out the ranking of the players.

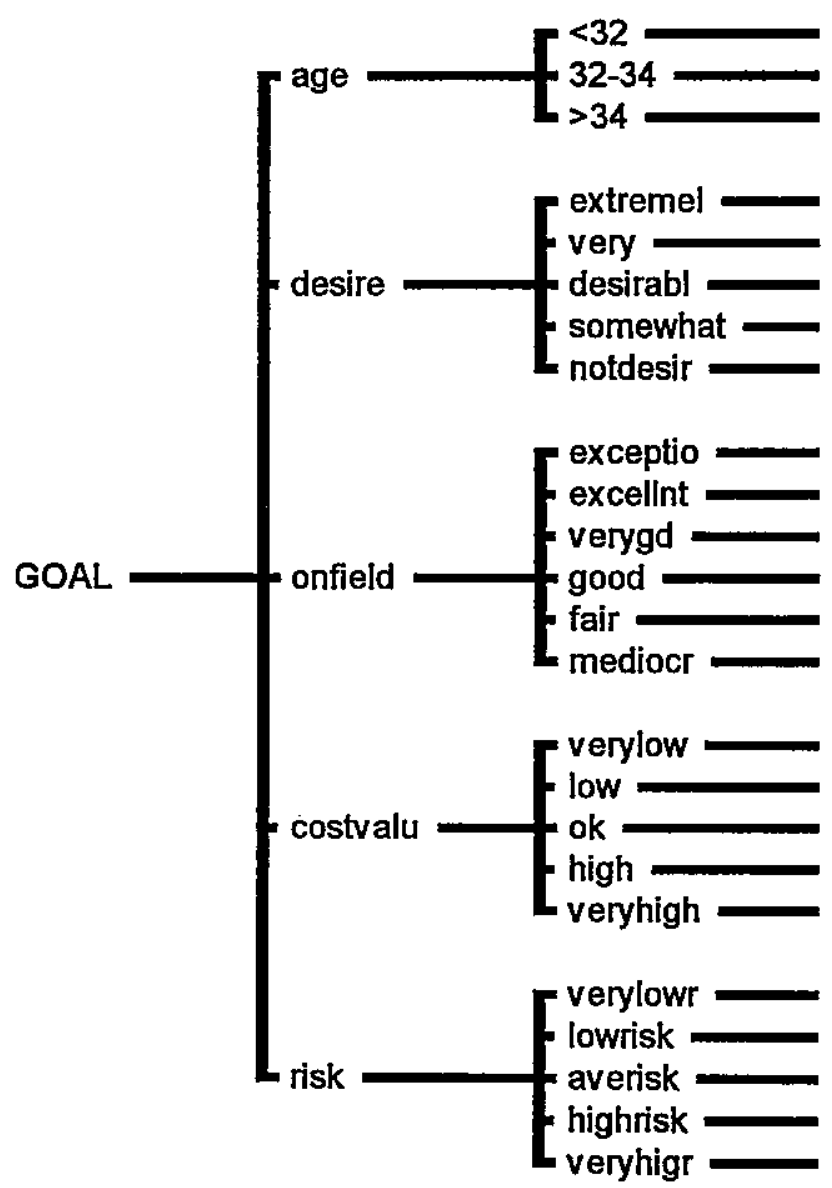

Figure 1: Sideway View of the AHP Tree 


\section{The AHP Criteria and Subcriteria}

We now describe the five major criteria, along with their subcriteria, that were used to rank the players. The mnemonic for each of these criteria, subcriteria and subsubcriteria are given in parentheses and denoted in bold. These mnemonics are used in the AHP tree presented in Figure 1.

AGE OF THE PLAYER (age). Almost all free agents are at least 30 years of age. Age can play a role in deciding upon the term (in years) of the contract to offer a free agent. In the model, the age of the player is broken down into the following three subcriteria: no older than 32 years $(<32)$, between 32 and 34 years old (32-34), and at least 35 years old $(>34)$.

DESIRABILITY OF THE PLAYER (desire). We attempted to assess how desirable this player is to the team doing the analysis. Desirability can reflect the importance of the player to the team or the player fills a specific need (for example, the team needs a catcher or a closer), The data, as we prepared it, was carried out for a generic team and our analysis did not consider the needs of a specific team. Thus, the desirability of a player who is a designated hitter only would be significantly higher for an American League team than a National League team. This criteria was then broken down into the following five subcriteria: extremely desirable (extremel), very desirable (very), desirable (desirabl), somewhat desirable (somewhat) and not desirable (notdesir). This criterion is a modification of the criterion suggested by John Sniezek in the model developed in Bodin and Epstein 1999.

ON-FIELD PERFORMANCE (onfield). Under this criterion, we tried to evaluate the player's anticipated on-field performance over the duration of the contract that the team might offer the player. Obviously, on-field performance becomes difficult to ascertain - especially for contracts at least four years in duration and the age of the player being in the mid 30's. In reality, therefore, this criterion became the anticipated on-field performance of the player of the next couple of years. The longer term performance was then factored into the risk of the player (the last category). This criterion was broken down into following subcriteria: exceptional on field performance (exceptio), excellent on field performance (excellnt), very good on field performance (verygd), good on field performance over the next two years (good), fair on field performance over the next two years (fair), and mediocre on field performance (mediocr). This criterion turned out to be the highest weighed criterion in our analysis, getting $50 \%$ of the weight.

COST TO VALUE RATIO (costvalu). The cost to value ratio of a player is the cost of a player to the anticipated value of the player over the term of the contract. In today's business of major league baseball, most free agents of any significant value sign contracts worth at least one million dollars a year. However, if the performance of a player who earns several million dollars a year is so exemplary, management may feel that this player is 'worth every dollar he earns.' In this case, the player will be given a rating of very low or low in this criterion. This criterion, which attempts to measure the marginal cost of a player over the term of the contract has the following five subcriteria: a very low cost to value ratio (verylow), a low cost to value ratio (low), a reasonable cost to value ratio (ok), a high cost to value ratio (high), and a very high cost to value ratio (very hig). As with the on-field performance criterion, this criterion is effectively measured over two years and not the duration of the contract. The teams know that in a long term contract, there can be diminishing returns far into the future. However, these team have such a high desire to win that they tend to mortgage the future and disregard the player's diminished capabilities further into the future.

RISK (risk). The risk of a player is the perceived risk in signing the player to the contract that the team wishes to offer the player. Risk can account for the anticipated performance of the player later in the term of the contract and the possibility of injury including the perceived health of the player at the time the player signs the contract. For example, David Cone was rated a very high risk and Randy Johnson was rated a high risk because of the possibility of Cone re-injuring his arm and Johnson re-injuring his back. Mo Vaughn was rated a average risk because of his size (over 240 pounds) offered the possibility of hurting himself (although he does not have a history of being 
injured). Risk was broken down into four subcriteria: very low risk (verylowr), low risk (lowrisk), average risk (averisk), high risk (highrisk), and very high risk (veryhigr).

\section{Analysis}

The above model was used to evaluate 72 of the 128 free agents that were available after the 1998 season. This analysis was carried out in November, 1998 before any free agents signed with a team. The other free agents were not evaluated because we did not have enough information on these free agents to do a discriminating evaluation and most of these free agents were not considered to be very desirable and, therefore, would not command a high salary or long term contract. The highest rated free agents in our evaluation is shown if Figure 2.

This model appeared to be an excellent predictor of the types of contract the free agents would receive. Any player that we rated exceptional or excellent on-field performance and very low or low cost-to-value ratio received contracts that was worth many millions of dollars a year. The term of the contract for these players was based on two factors - their risk and their age. If the player had a higher risk and was over 34, then the term of the contract was less. David Cone and Ken Caminiti were two examples of this situation. Both players have been severely injured in the past couple of years. Because of that, Cone received a one year contract (at $\$ 9$ million/year) whereas Caminiti received a two year $\$ 11$ million contract (approximately $\$ 5.5$ million a year) although it was known that Caminiti was willing to sign a smaller contract to play in Houston. Another player that falls into this situation is Ramon Martinez. Martinez's contract initially paid a little amount of money but contained many incentives (based on statistics such as number of wins or innings pitched). The Red Sox have an option to sign Martinez for the third year. Martinez cand earn about $\$ 24$ million in 3 years. However, the guarantee to Martinez is quite small.

How have these higher risk players worked out two months into the 1999 season. As of May 27, 1999, Cone and Johnson are pitching exceptionally well with no injury problems. Vaughn is hitting well now but was not able to pay because of a leg injury for a few weeks in April. Vaughn was hurt when he fell into the dugout attempting to catch a foul ball in the first game of the season. Caminiti has played well but is now injured and on the disabled list. Martinez has not pitched an inning as yet this year and is due back in the middle of July. The Red Sox knew that Martinez was injured when they signed him. For them, paying Martinez for over half a season while he was recuperating was an investment for the productivity that they would receive in the remainder of his contract.

Injury risk is a major consideration in signing free agents. How much weight the user should put on risk needs to be considered carefully in using this model. I know from playing a considerable amount of rotisserie baseball that my partner and I carefully consider injury risk in deciding upon the players to select. An injury to a key player on the team can devastate the team and render the team noncompetitive for that year. I believe that teams should not invest a great amount of their resources in players who are injury prone or near the end of their career. I believe a better strategy is for a team to trade for these players in July when the season is $2 / 3$ rds complete and you know that the players are healthy rather than assume the risk at the beginning of the season. These teams only have these players for a short term. Trades in late July are made by teams contending for the playoffs and these teams need additional productivity the last 60 games of the season. As reflected in our ratings, we believe that teams should adopt a more risk-averse strategy in the signing of free agents in the off-season. They should only sign high risk players if the term of the contract is short, the contract does not have a lot of money up-front but offers the player many incentives. The Ramon Martinez contract may be considered a prototype contract in this regard.

\section{References}

Bodin, L. and E. Epstein (1999), "Who on First with Probability .4), Computers and Operations Research, to appear. 
C:LECWINLFREAGENT

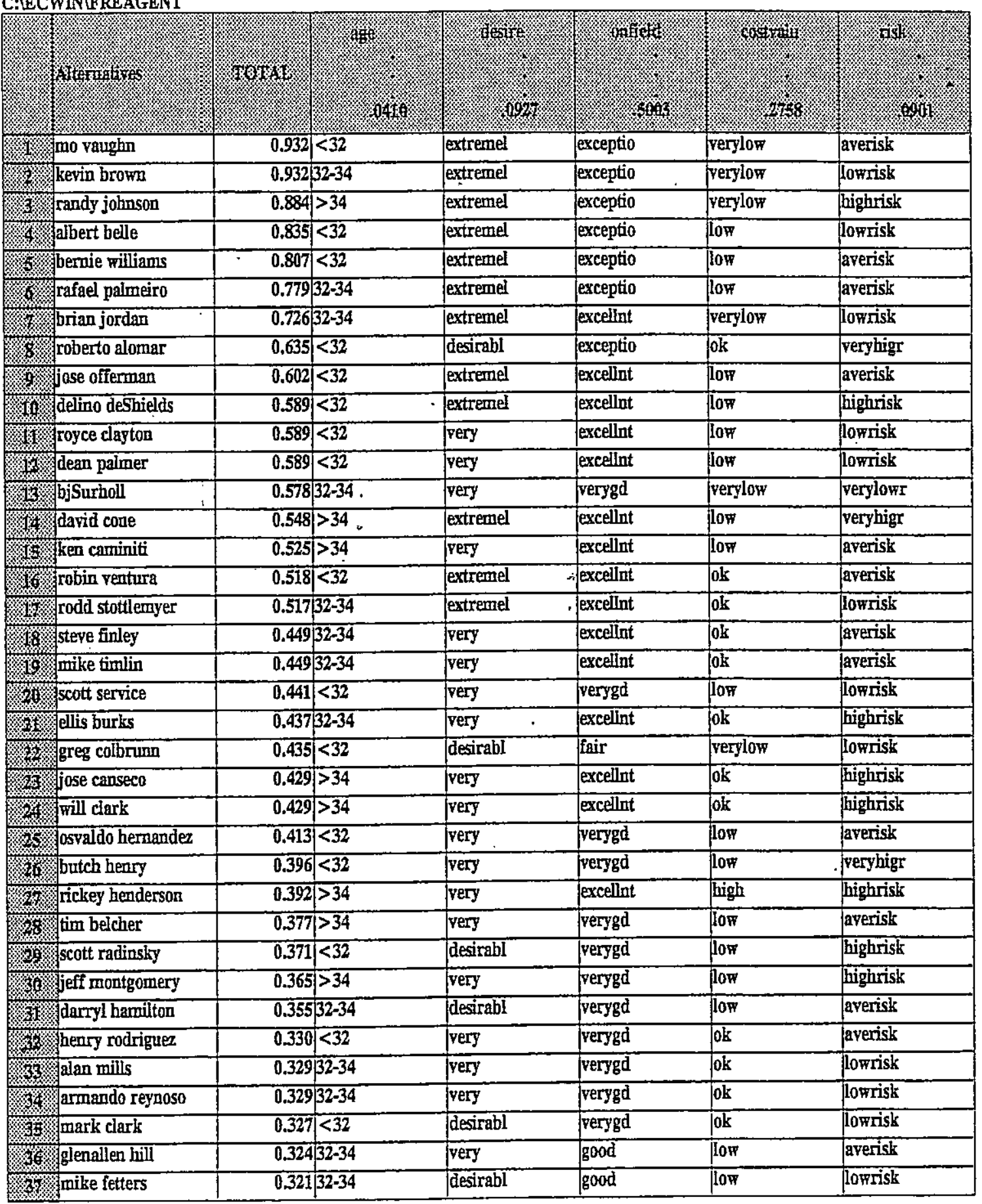

Figure 2: $\quad$ Ratings of the Top 40 Free Agents in the 1998 Expansion Draft 